\title{
Identification of differentially expressed genes, associated functional terms pathways, and candidate diagnostic biomarkers in inflammatory bowel diseases by bioinformatics analysis
}

\author{
CHUNWEI CHENG ${ }^{1}$, JUAN HUA $^{2}$, JUN TAN $^{1}$, WEI QIAN ${ }^{1}$, LEI ZHANG ${ }^{1}$ and XIAOHUA HOU ${ }^{1}$ \\ ${ }^{1}$ Division of Gastroenterology, Union Hospital, Tongji Medical College, Huazhong University of Science \\ and Technology, Wuhan, Hubei 430022; ${ }^{2}$ Department of Cardiology, The Sixth Hospital of Wuhan, \\ Affiliated Hospital of Jianghan University, Wuhan, Hubei 430015, P.R. China
}

Received September 27, 2018; Accepted March 29, 2019

DOI: $10.3892 /$ etm.2019.7541

\begin{abstract}
Inflammatory bowel diseases (IBDs), including ulcerative colitis (UC) and Crohn's disease (CD), are chronic inflammatory disorders caused by genetic influences, the immune system and environmental factors. However, the underlying pathogenesis of IBDs and the pivotal molecular interactions remain to be fully elucidated. The aim of the present study was to identify genetic signatures in patients with IBDs and elucidate the potential molecular mechanisms underlying IBD subtypes. The gene expression profiles of the GSE75214 datasets were obtained from the Gene Expression Omnibus database. Differentially expressed genes (DEGs) were identified in UC and CD patients compared with controls using the GEO2R tool. Gene Ontology (GO) and Kyoto Encyclopedia of Genes and Genomes (KEGG) pathway analyses of DEGs were performed using DAVID. Furthermore, protein-protein interaction (PPI) networks of the DEGs were constructed using Cytoscape software. Subsequently, significant modules were selected and the hub genes were identified. In the GO and KEGG pathway analysis, the top enriched pathways in UC and CD included Staphylococcus aureus infection, rheumatoid arthritis, complement and coagulation cascades, PI3K/Akt signaling pathway and osteoclast differentiation. In addition, the GO terms in the category biological process significantly enriched by these genes were inflammatory response, immune response, leukocyte migration, cell adhesion, response to
\end{abstract}

Correspondence to: Professor Xiaohua Hou, Division of Gastroenterology, Union Hospital, Tongji Medical College, Huazhong University of Science and Technology, 1277 Jiefang Avenue, Wuhan, Hubei 430022, P.R. China

E-mail: houxhmed@163.com

Key words: bioinformatics analysis, inflammatory bowel disease, ulcerative colitis, Crohn's disease, differentially expressed genes, protein-protein interaction network, hub genes, module analysis molecules of bacterial origin and extracellular matrix (ECM) organization. However, several other biological processes (GO terms) and pathways (e.g., 'chemotaxis', 'collagen catabolic process' and 'ECM-receptor interaction') exhibited significant differences between the two subtypes of IBD. The top 10 hub genes were identified from the PPI network using respective DEGs. Of note, the hub genes $G$ protein subunit gamma 11 (GNG11), G protein subunit beta 4 (GNB4), Angiotensinogen (AGT), Phosphoinositide-3-kinase regulatory subunit 3 (PIK3R3) and C-C motif chemokine receptor 7 (CCR7) are disease-specific and may be used as biomarkers for differentiating UC from CD. Furthermore, module analysis further confirmed that common significant pathways involved in the pathogenesis of IBD subtypes were associated with chemokine-induced inflammation, innate immunity, adapted immunity and infectious microbes. In conclusion, the present study identified DEGs, key target genes, functional pathways and enrichment analysis of IBDs, enhancing the understanding of the pathogenesis of IBDs and also advancing the clarification of the underlying molecular mechanisms of UC and CD. Furthermore, these results may provide potential molecular targets and diagnostic biomarkers for UC and CD.

\section{Introduction}

Inflammatory bowel diseases (IBDs) are chronic idiopathic inflammatory disorders of the gastrointestinal tract with two distinct phenotypes: Ulcerative colitis (UC) and Crohn's disease (CD) $(1,2)$. UC is characterized by relapsing and remitting mucosal inflammation extending from the rectum to proximal segments of the colon (2). By contrast, CD may affect the entire digestive tract and cause transmural inflammation (3). The prevalence of IBDs is increasing steadily in Western countries, and developing countries have had a rapidly increasing incidence in recent years $(4,5)$. For instance, the incidence of IBDs in China has reached 3.3 per 100,000 and exhibits a further increasing trend (6). Furthermore, IBD frequently progresses into a long-term 
health condition with a variety of complications and reduces patients' health-associated quality of life $(7,8)$. Therefore, it is essential to expand the current understanding of the pathogenesis of IBDs to develop effective drugs and therapies for its treatment.

Despite great advances toward the understanding of the pathophysiology of IBDs, early diagnosis, therapeutic intervention and the underlying molecular mechanisms of IBD remain a challenge. Furthermore, in spite of certain differences, $\mathrm{UC}$ and $\mathrm{CD}$ share overlapping genetic and clinical features, making it challenging to diagnose and distinguish between them. Therefore, elucidation of the unique characteristics of $\mathrm{UC}$ or CD is also vital for the development of more precise diagnostic and effective therapeutic strategies to improve patient outcome.

Recently, with the continuous development of bioinformatics and molecular biology, microarray technology has been widely used for exploring the molecular mechanisms of various diseases (9-11). In previous decades, analysis of the expression profiles of gene microarrays was used to identify several key genes and diagnostic biomarkers of IBDs, including several differentially expressed genes (DEGs) involved in different pathways, biological processes or molecular functions $(12,13)$. Furthermore, several key genes and candidate biomarkers of IBDs, including Ring finger protein 186, Cadherin 11, Intercellular adhesion molecule 1 (ICAM1) and Hepatocyte nuclear factor 4 alpha, have been identified by bioinformatics analyses (14-17). However, previous studies have mainly focused on identifying candidate genes for UC or CD, while the potential intrinsic links among DEGs have not been extensively investigated.

In the present study, a gene expression dataset (GSE75214) was downloaded from the Gene Expression Omnibus (GEO) database. The DEGs were identified in UC patients and CD patients compared with controls, respectively. Gene Ontology (GO) and Kyoto Encyclopedia of Genes and Genomes (KEGG) analyses performed in the DAVID database were applied to determine the functional enrichment and significant pathways associated with the DEGs. In addition, a protein-protein interaction (PPI) network of common DEGs was constructed to identify the critical genes and significant modules. By means of analyzing of the biological functions and pathways shared between and unique to UC and CD, the present study provided further insight into the pathogenesis of UC and CD at the molecular level, which may facilitate the diagnosis and provide potential molecular targets for UC and CD.

\section{Materials and methods}

Microarray data. The GEO database (https://www.ncbi.nlm. nih.gov/geo/) stores original submitter-supplied records as well as curated datasets. The gene expression dataset GSE75214 was obtained from the GEO database. This dataset had been generated using the platform GPL6244 \{(HuGene-1_0-st) Affymetrix Human Gene 1.0 ST Array [transcript (gene) version]\} and was deposited by Vancamelbeke et al (18) in the GEO database. A total of 194 samples are included in this dataset, comprising colon tissues from 97 patients with UC (74 with active disease and 23 with inactive disease),
34 patients with CD (8 with active colonic disease and 26with inactive disease), and 11 controls, as well as the (neo-) terminal ileum of $67 \mathrm{CD}$ patients and 11 controls. The baseline characteristic data of the subjects was published previously by Vancamelbeke et al (18). The present study focused on colonic mucosal biopsies (74 active UC, 8 active CD patients and 11 controls) and excluded the ileal biopsies. Thus, a total of 93 chips were available for the subsequent analysis.

Identification of DEGs. Data pre-processing was performed using GEO2R (https://www.ncbi.nlm.nih.gov/geo/geo2r/) and was applied to screen DEGs between the following groups: UC vs. Controls and CD vs. Controls. GEO2R is an interactive web tool that compares two groups of samples under the same experimental conditions and is able to analyze almost any GEO series (https://www.ncbi. nlm.nih.gov/geo/geo2r/). The adjusted $\mathrm{P}<0.01$ and $\log _{2}$ fold change (FC) $\mid>1$ (i.e., FC $>2$ ) were selected as the threshold for each group.

GO and KEGG pathway enrichment analyses. The Database for Annotation, Visualization and Integrated Discovery (DAVID 6.8, https://david.ncifcrf.gov/summary.jsp) is an online program that may be used to systematically extract biological meaning from large lists of genes or proteins. In order to analyze the screened DEGs at the functional level, GO enrichment and KEGG pathway analysis was performed using the DAVID online tool. $\mathrm{P}<0.05$ was set as the cut-off criterion.

PPI network analysis. To further evaluate the functional interactions among DEGs, a PPI network was used. The DEGs were mapped using the Search Tool for the Retrieval of Interacting Genes (STRING; version 11.0; https://string-db. org/cgi/input.pl) and only the interaction pairs with a PPI combined score of $>0.7$ were selected as significant. Subsequently, the PPI network was constructed using Cytoscape software (https://cytoscape.org/; version 3.6.1). The top 10 essential nodes ranked in the network of respective DEGs were calculated using the Cytoscape plugin CytoHubba.

Module analysis. The plug-in Molecular Complex Detection (MCODE) was used to identify the densely connected regions of the PPI network in Cytoscape. MCODE was then applied to screen modules of the PPI network with the following parameters: Degree cutoff, 2; node score cutoff, 0.2; k-core, 2; and maximum depth, 100. In addition, the functions and pathways of the DEGs in each module were assessed using ClueGo, with $\mathrm{P}<0.05$ being considered to indicate significance.

\section{Results}

Identification of DEGs. The gene expression dataset GSE75214 was downloaded from the GEO database. DEGs between the disease samples and the controls were determined using the GEO2R tool. As presented in Fig. 1, a total of 1,236 DEGs were identified in the UC group using the threshold of $\mathrm{P}<0.05$ 
Table I. Top 10 up- and downregulated differentially expressed genes in ulcerative colitis vs. control.

\begin{tabular}{lcc}
\hline Gene symbol & Log fold-change & Adjusted P-value \\
\hline Upregulated & & \\
SLC6A14 & 5.72 & $3.96 \times 10^{-23}$ \\
MMP3 & 4.80 & $1.09 \times 10^{-12}$ \\
REG1A & 4.63 & $1.61 \times 10^{-10}$ \\
DUOX2 & 4.34 & $1.31 \times 10^{-15}$ \\
TINP3 & 4.32 & $2.15 \times 10^{-16}$ \\
DUOXA2 & 4.32 & $2.84 \times 10^{-18}$ \\
IDO1 & 4.23 & $2.77 \times 10^{-16}$ \\
S100A8 & 4.15 & $1.01 \times 10^{-11}$ \\
MMP1 & 4.14 & $5.77 \times 10^{-11}$ \\
REG1B & 4.08 & $4.31 \times 10^{-7}$ \\
Downregulated & & \\
AQP8 & -5.52 & $8.44 \times 10^{-24}$ \\
HMGCS2 & -4.76 & $5.65 \times 10^{-17}$ \\
SLC26A2 & -4.52 & $1.45 \times 10^{-19}$ \\
CLDN8 & -3.85 & $2.95 \times 10^{-15}$ \\
ABCG2 & -3.80 & $3.77 \times 10^{-19}$ \\
PCK1 & -3.71 & $6.10 \times 10^{-15}$ \\
TMIGD1 & -3.71 & $7.83 \times 10^{-11}$ \\
OTOP2 & -3.37 & $2.22 \times 10^{-16}$ \\
GUCA2A & -3.32 & $1.02 \times 10^{-9}$ \\
UGT2A3 & -3.24 & $2.04 \times 10^{-26}$ \\
\hline
\end{tabular}

and $\log _{2} \mathrm{FCl}>1$, including 790 upregulated genes and 446 downregulated genes in the UC vs. control samples. The top 10 up- and downregulated genes for UC vs. the control are listed in Table I.

Similarly, comparison of the CD group with the healthy control samples identified 588 DEGs, including 438 upregulated and 150 downregulated genes. The top 10 DEGs for CD vs. control samples are listed in Table II. Of note, most of the DEGs identified in UC were also differentially expressed in CD. A total of 565 overlapping DEGs were identified between UC and CD vs. the controls (Fig. 1). Thus, a total of 671 and 23 specific DEGs remained for $\mathrm{UC}$ and $\mathrm{CD}$, respectively (Fig. 1).

GO and pathway enrichment analysis of DEGs. To further perform a systematic characterization and explore the biological functions of the identified DEGs in UC and CD vs. control samples, functional annotation and pathway analyses, including GO and KEGG analyses, were performed using DAVID. The top $10 \mathrm{GO}$ terms in the category biological process are presented in Fig. 2. The results of the GO analysis indicated that most of the DEGs in the UC and CD groups were simultaneously enriched in inflammatory response, immune response, leukocyte migration, response to lipopolysaccharides, extracellular matrix organization, cell adhesion and positive regulation of angiogenesis (Fig. 2A). In addition, several DEGs in UC were enriched in chemotaxis, cell chemotaxis and cell surface receptor signaling pathways (Fig. 2A).
Table II. Top 10 up- and downregulated differentially expressed genes in Crohn's disease vs. control.

\begin{tabular}{lcc}
\hline Gene symbol & Log fold-change & Adjusted P-value \\
\hline Upregulated & & \\
SLC6A14 & 4.77 & $2.34 \times 10^{-7}$ \\
REG1A & 4.68 & $1.12 \times 10^{-3}$ \\
MMP3 & 4.64 & $1.59 \times 10^{-4}$ \\
REG1B & 4.29 & $1.28 \times 10^{-3}$ \\
MMP1 & 4.28 & $3.91 \times 10^{-4}$ \\
S100A8 & 4.24 & $1.29 \times 10^{-5}$ \\
IDO1 & 4.13 & $1.17 \times 10^{-6}$ \\
DUOX2 & 3.95 & $1.25 \times 10^{-4}$ \\
CHI3L1 & 3.73 & $5.93 \times 10^{-6}$ \\
REG3A & 3.63 & $3.53 \times 10^{-3}$ \\
Downregulated & & \\
AQP8 & -3.47 & $6.29 \times 10^{-4}$ \\
ABCG2 & -3.05 & $4.30 \times 10^{-5}$ \\
CYP2B7P & -2.86 & $2.66 \times 10^{-5}$ \\
HMGCS2 & -2.69 & $1.36 \mathrm{Ex} 10^{-3}$ \\
SLC30A10 & -2.51 & $2.34 \times 10^{-4}$ \\
PRKG2 & -2.48 & $2.52 \times 10^{-4}$ \\
CYP2B6 & -2.44 & $1.33 \times 10^{-3}$ \\
SLC16A9 & -2.40 & $1.03 \times 10^{-4}$ \\
ABCB1 & -2.28 & $5.02 \times 10^{-5}$ \\
APOBEC3B & -2.26 & $5.99 \times 10^{-6}$ \\
\hline
\end{tabular}

Similarly, certain DEGs in the CD group were also closely associated with defense response to viruses and collagen catabolic process (Fig. 2B).

Subsequently, KEGG pathway analysis indicated that the DEGs in UC and CD were primarily enriched in Staphylococcus aureus infection, rheumatoid arthritis, complement and coagulation cascades, malaria, leishmaniasis, the PI3K/Akt signaling pathway and osteoclast differentiation (Fig. 2A and B). In addition, certain KEGG pathways, including cytokine-cytokine receptor interaction, cell adhesion molecules and chemokine signaling pathway, were commonly involved in the development of UC, whereas certain other pathways [extracellular matrix (ECM)-receptor interaction, tumor necrosis factor (TNF) signaling pathway and pertussis] were mainly involved in the pathogenesis of $\mathrm{CD}$ (Fig. 2A and B). Therefore, several GO terms in the category biological process and KEGG pathways exhibited significant differences between $\mathrm{UC}$ and $\mathrm{CD}$, indicating that $\mathrm{UC}$ and $\mathrm{CD}$ have different pathological characteristics.

Construction of PPI network and identification of hub genes. To further explore the association between DEGs at the protein level, the PPI networks were constructed based on the interactions of DEGs. DEGs for UC and CD, as well as their overlapping DEGs, were mapped to PPI networks and visualized by Cytoscape software, respectively. With the pre-defined criterion of a combined score of $>0.7$, the PPI network of the overlapping DEGs consisted of 307 nodes 
A

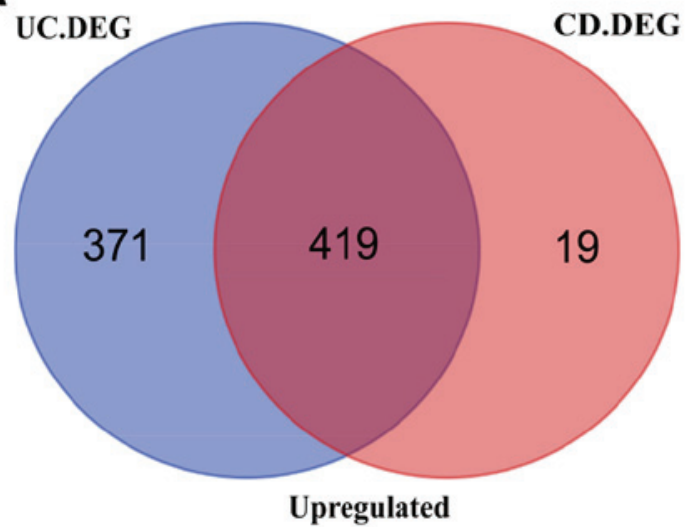

B

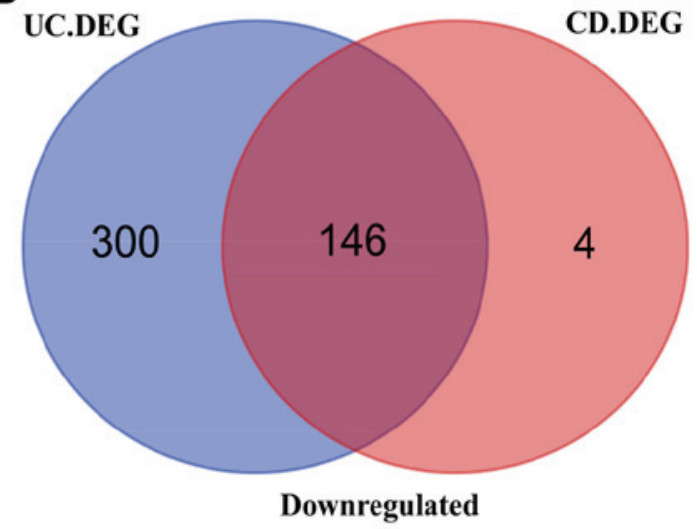

Figure 1. Venn diagrams illustrating the number of (A) upregulated and (B) downregulated genes in UC and CD. The intersection represents the DEGs shared between the two groups. UC, ulcerative colitis; $\mathrm{CD}$, Crohn's disease; DEG, differentially expressed gene.

\section{A Biological process}

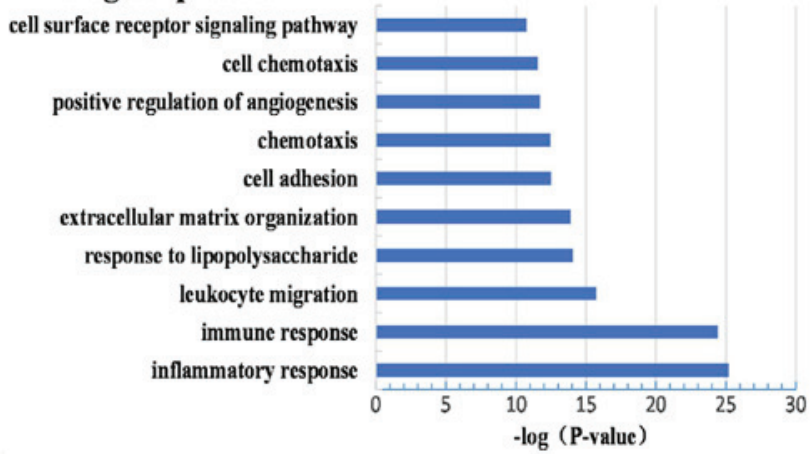

\section{B Biological process}

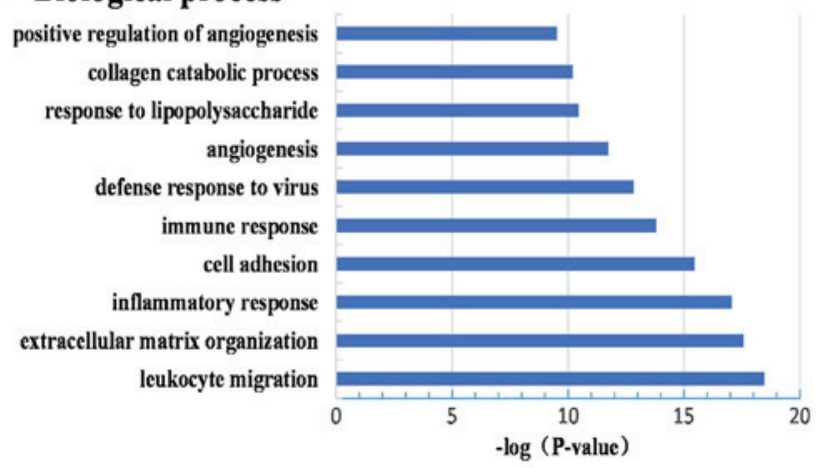

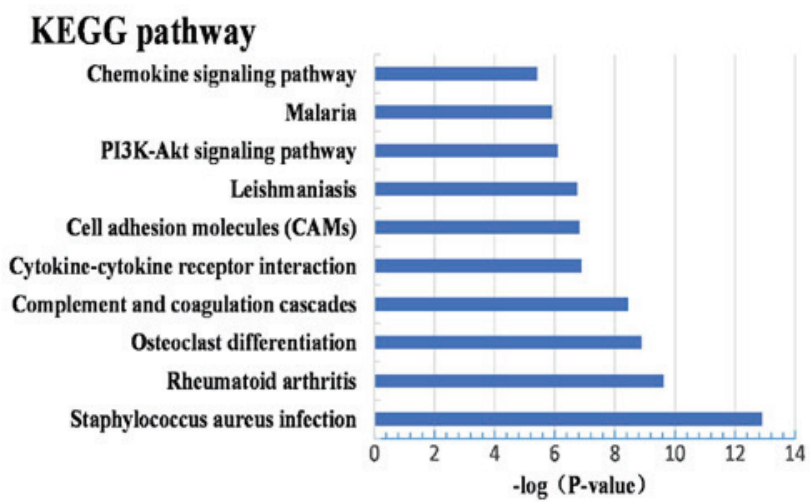

KEGG pathway

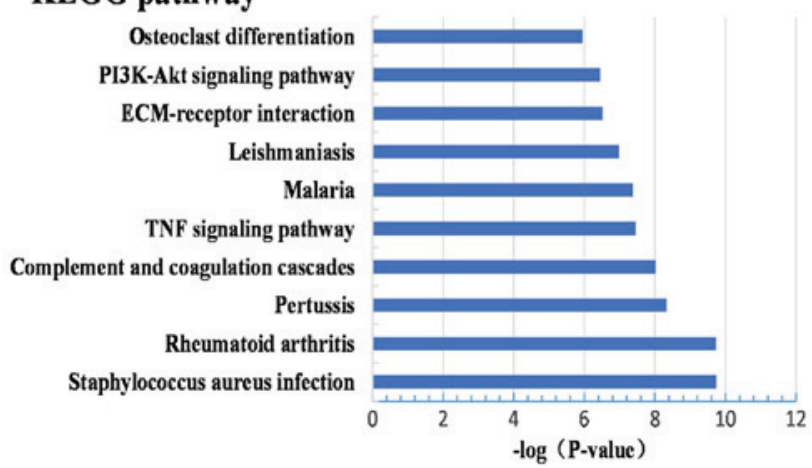

Figure 2. GO and KEGG pathway functional enrichment analysis of significant DEGs in (A) the ulcerative colitis group and in (B) the Crohn's disease group (top $10 \mathrm{GO}$ terms of biological processes and functional pathways). The vertical axis represents the GO term or KEGG pathway terms significantly enriched by the DEGs; the horizontal axis indicates the negative Log10 (P-value). GO, Gene Ontology; DEG, differentially expressed gene.

and 1,205 edges. The top 10 hub nodes with a high degree of interaction in this network are presented in Table III. These hub genes were interleukin Interleukin 6 (IL6), cluster of differentiation 44, IL8, IL1B, ICAM1, Signal transducer and activator of transcription 1 (STAT1), Jun proto-oncogene, Transforming growth factor beta 1, Janus kinase 2 (JAK2) and Matrix metallopeptidase 2 (MMP2), which were identified as the candidate genes. Similarly, the PPI networks for UC and CD individually were constructed, including 631 and 312 nodes, and 3,231 and 1,218 edges, respectively. Among these nodes, the top 10 hub genes with the highest degree of interaction are provided in Table III. Of note, G protein subunit gamma 11 (GNG11), G protein subunit beta 4 (GNB4), Angiotensinogen (AGT), Phosphoinositide-3-kinase regulatory subunit 3 (PIK3R3) and $\mathrm{C}-\mathrm{C}$ motif chemokine receptor 7 (CCR7) were highly expressed in UC but not in CD. These specific hub genes may be regarded as candidate marker genes for UC. However, the hub genes for CD overlapped with the DEGs for UC, and there were no specific hub genes for $\mathrm{CD}$. Most of the hub genes in CD were mainly associated with the chemokine signaling pathway, leukocyte migration and intestinal fibrosis-associated pathways. Several of the 
A

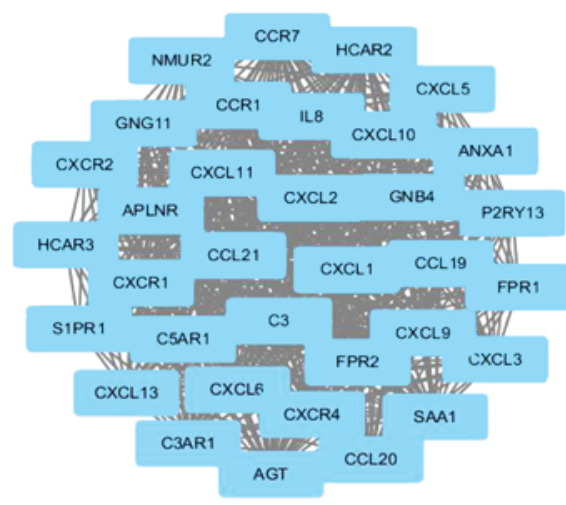

B

\begin{tabular}{|c|c|c|c|}
\hline Pathway term & Gene count & P-value & Genes \\
\hline $\begin{array}{l}\text { Chemokine signaling } \\
\text { pathway }\end{array}$ & 19 & $1.55 \times 10^{-21}$ & $\begin{array}{l}\text { CXCL1, CXCL5, CXCL3, CCR1, } \\
\text { CXCL2, CXCL9, CXCR1, CCL19, } \\
\text { CXCR2, GNG11, CXCL6, CXCL11, } \\
\text { CXCL10, CCR7, CCL20, CXCR4, } \\
\text { CCL21, CXCL13, GNB4 }\end{array}$ \\
\hline $\begin{array}{l}\text { Cytokine-cytokine } \\
\text { receptor interaction }\end{array}$ & 12 & $1.37 \times 10^{-9}$ & $\begin{array}{l}\text { CCR7, CCL20, CXCR4, CXCL13, } \\
\text { CCL21, CCR1, CXCL9, CXCR1, } \\
\text { CCL19, CXCR2, CXCL11, CXCL10 }\end{array}$ \\
\hline $\begin{array}{l}\text { Staphylococcus aureus } \\
\text { infection }\end{array}$ & 5 & $7.85 \times 10^{-5}$ & C3AR1, C5AR1, C3, FPR1, FPR2 \\
\hline $\begin{array}{l}\text { Neuroactive ligand- } \\
\text { receptor interaction }\end{array}$ & 8 & $1.42 \times 10^{-4}$ & $\begin{array}{l}\text { P2RY13, C3AR1, APLNR, S1PR1, } \\
\text { C5AR1, NMUR2, FPR1, FPR2 }\end{array}$ \\
\hline TNF signaling pathway & 5 & $1.05 \times 10^{-3}$ & $\begin{array}{l}\text { CXCL1, CCL20, CXCL3, CXCL2, } \\
\text { CXCL10 }\end{array}$ \\
\hline Legionellosis & 4 & $1.58 \times 10^{-3}$ & CXCL1, C3, CXCL3, CXCL2 \\
\hline $\begin{array}{l}\text { Complement and } \\
\text { coagulation cascades }\end{array}$ & 3 & $3.57 \times 10^{-2}$ & C3AR1, C5AR1, C3 \\
\hline Pertussis & 3 & $4.16 \times 10^{-2}$ & CXCL5, C3, CXCL6 \\
\hline
\end{tabular}

Figure 3. The top module of the protein-protein interaction network of the differentially expressed genes for UC vs. controls. (A) The top module of UC. (B) The enriched pathways of the top module for UC. UC, ulcerative colitis.

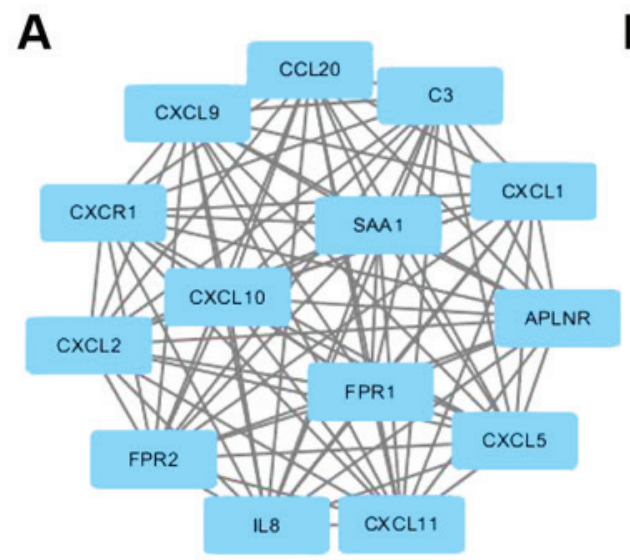

B

\begin{tabular}{lccl}
\hline Pathway term & Gene count & P-value & Genes \\
\hline $\begin{array}{l}\text { Chemokine signaling } \\
\text { pathway }\end{array}$ & 8 & $2.76 \times 10^{-9}$ & $\begin{array}{l}\text { CXCL1, CXCL5, CCL20, CXCL2, } \\
\text { CXCL9, CXCR1, CXCL11, CXCL10 }\end{array}$ \\
$\begin{array}{l}\text { Cytokine-cytokine } \\
\text { receptor interaction }\end{array}$ & 5 & $3.28 \times 10^{-4}$ & $\begin{array}{l}\text { CCL20, CXCL9, CXCR1, CXCL11, } \\
\text { CXCL10 }\end{array}$ \\
$\begin{array}{l}\text { TNF signaling pathway } \\
\begin{array}{l}\text { Staphylococcus aureus } \\
\text { infection }\end{array}\end{array}$ & 4 & $5.29 \times 10^{-4}$ & CXCL1, CCL20, CXCL2, CXCL10 \\
$\begin{array}{l}\text { Legionellosis } \\
\begin{array}{l}\text { Toll-like receptor } \\
\text { signaling pathway }\end{array}\end{array}$ & 3 & $3.15 \times 10^{-3}$ & C3, FPR1, FPR2 \\
& 3 & $3.15 \times 10^{-3}$ & CXCL1, C3, CXCL2 \\
\hline
\end{tabular}

Figure 4. The top module of the protein-protein interaction network of the differentially expressed genes for CD vs. controls. (A) The top module of CD. (B) The enriched pathways of the top module for CD. CD, Crohn's disease.

candidate genes identified may be used as biomarkers for the differential diagnosis of CD and UC. The hub genes, GNG11 and GNB4, that were identified in UC tissues may therefore serve to distinguish UC from CD. However, certain candidate genes, including JAK2 and MMP2, that were identified in CD tissues may not differentiate UC from CD.

Module analysis of the PPI network. With the application of the MCODE plugin of Cytoscape, the top modules of the PPI networks for UC and CD were selected, and a functional enrichment analysis of DEGs in the respective functional modules was (Figs. 3-5). KEGG pathway enrichment analysis revealed that the DEGs in UC and CD in the modules were primarily associated with the chemokine signaling pathway, cytokine-cytokine receptor interaction,
Staphylococcus aureus infection, TNF signaling pathway and legionellosis (Figs. 3 and 4).

While UC and CD are different diseases, they have various pathological characteristics in common; therefore, the biological functions and pathways shared between UC and CD were then analyzed. A total of 565 overlapped DEGs were identified for the UC and CD groups. Subsequently, one significant module was obtained from the PPI network of the overlapped DEGs (Fig. 5). The results of the GO analysis indicated that genes in the top module were primarily associated with cell chemotaxis, killing of cells of other organisms and positive chemotaxis (Fig. 5). KEGG pathway enrichment analysis of the DEGs included in the top module revealed that these genes were mainly associated with chemokine signaling pathway, Staphylococcus aureus infection, legionellosis and 
Table III. Top ten genes with the highest degree of interaction in the protein-protein interaction network using respective DEGs for UC, CD and UC + CD vs. controls.

\begin{tabular}{|c|c|}
\hline DEG & Degree of interaction \\
\hline \multicolumn{2}{|l|}{$\mathrm{UC}$} \\
\hline IL6 & 88 \\
\hline IL8 & 67 \\
\hline GNB4 & 62 \\
\hline GNG11 & 62 \\
\hline AGT & 59 \\
\hline CXCR4 & 54 \\
\hline ANXA1 & 54 \\
\hline PIK3R3 & 53 \\
\hline SAA1 & 52 \\
\hline CCR7 & 51 \\
\hline \multicolumn{2}{|l|}{$\mathrm{CD}$} \\
\hline IL6 & 63 \\
\hline CD44 & 39 \\
\hline IL8 & 37 \\
\hline IL1B & 36 \\
\hline ICAM1 & 34 \\
\hline STAT1 & 33 \\
\hline TGFB 1 & 30 \\
\hline JAK2 & 29 \\
\hline VCAM1 & 28 \\
\hline $\mathrm{C} 3$ & 27 \\
\hline \multicolumn{2}{|c|}{$\mathrm{UC}$ and $\mathrm{CD}$} \\
\hline IL6 & 63 \\
\hline CD44 & 39 \\
\hline IL8 & 38 \\
\hline IL1B & 36 \\
\hline ICAM1 & 34 \\
\hline STAT1 & 32 \\
\hline JUN & 31 \\
\hline TGFB 1 & 31 \\
\hline JAK2 & 29 \\
\hline MMP2 & 28 \\
\hline
\end{tabular}

UC, ulcerative colitis; CD, Crohn's disease; DEG, differentially expressed gene.

epithelial cell signaling in Helicobacter pylori infection (Fig. 6).

\section{Discussion}

$\mathrm{UC}$ and $\mathrm{CD}$ are the two major types of inflammatory bowel disease, whose etiology involves a complex interaction between genetics, environmental factors, infectious microbes and dysregulated immune responses (19). Although research on IBDs has achieved great progress in the past decade, the pathogenesis of IBDs has yet to be fully elucidated due to the complex and poorly understood interactions among these factors. Therefore, advancement of the understanding of the molecular mechanisms of IBDs based on microarray technology, which has developed rapidly and has been widely used to examine the pathogenic processes of various diseases (20-22), may provide potential diagnostic and therapeutic targets for UC and CD.

In the present study, 1,236 DEGs were identified in the UC group, including 790 upregulated and 446 downregulated genes, and in the CD group, 588 DEGs, including 438 upregulated and 150 downregulated genes, were identified. The UC and CD groups had 565 DEGs in common, indicating that the two disorders have an important and overlapping genetic component. GO analysis indicated that the identified DEGs for $\mathrm{UC}$ and $\mathrm{CD}$ are accumulated in similar functional terms in the category biological process, including inflammatory response, immune response, leukocyte migration, response to lipopolysaccharide, ECM organization and cell adhesion. Previous studies have demonstrated that the aforementioned GO terms are potentially important events in the pathogenesis of IBDs. For instance, immuno-inflammatory responses, leukocyte migration, cell chemotaxis and cell adhesion have roles in the pathogenesis of IBDs $(19,23,24)$. In addition, accumulating evidence has demonstrated that angiogenesis has a pathogenic role in IBDs (25-27). Growth of new blood vessels is an important component of the inflammatory process, and exacerbation of inflammation in a vicious circle that aggravates mucosal damage and remodeling (26). Therefore, several significant DEGs of the angiogenesis pathway may be used as therapeutic targets for adjuvant therapy.

Furthermore, KEGG pathway analysis revealed that UC and CD also shared the top enriched pathways, including Staphylococcus aureus infection, rheumatoid arthritis, complement and coagulation cascades, PI3K/Akt signaling pathway and osteoclast differentiation. Previous studies have demonstrated that infectious microbes have role in the development of IBDs. The present study indicated that UC as well as CD were associated with Staphylococcus aureus infection, malaria and leishmaniasis. Hu and Agarwal (28) reported that malaria drugs may be used to treat $\mathrm{CD}$, indicating a potential association between malaria and IBDs. In addition, a number of extra-intestinal diseases have been associated with IBD. For instance, patients with IBD were reported to have an increased prevalence of rheumatoid arthritis (29-31). Previous studies have also demonstrated that patients with IBDs have an increased risk of developing osteopenia and osteoporosis, which is a common complication of IBDs and associated with an impaired quality of life $(32,33)$. Ciucci et al $(34)$ reported that $\mathrm{CD}^{+} \mathrm{T}$-cell subsets contributed to the differentiation of osteoclasts and maintained a vicious circle linking inflammation and bone destruction. The PI3K/Akt pathway is associated with cell cycle progression, cell death and cell growth (35). Recent studies have reported that the PI3K/Akt pathway is involved in the pathogenesis of IBDs by exerting pro-inflammatory effects and activating $\mathrm{T}$ cells (36-38). In the present study, several GO terms in the category biological process and KEGG pathways exhibited significant differences between UC and CD, including chemotaxis, collagen catabolic process, cytokine-cytokine receptor interaction and ECM-receptor interaction. Previous studies have demonstrated that intestinal fibrosis with stricture formation is a 
A

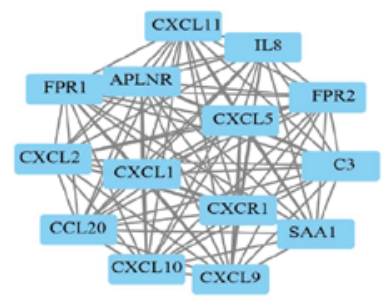

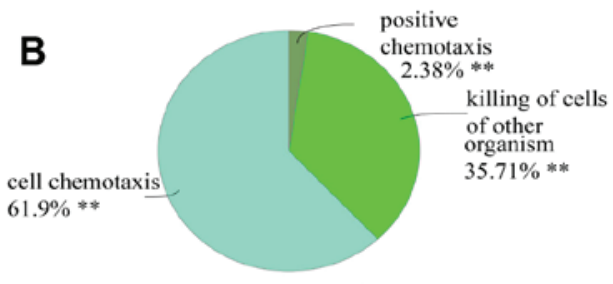

$\%$ Genes / Term

C positive chemotaxis positive regulation of release of sequester... killing of cells of other organism antimicrobial humoral response
disruption of cells of other organism lymphocyte migration
mune response mediat... antimicrobial humoral immune response mediat...
monocyte chemotaxis
lymphocyte chemotaxis
T cell migration
$T$ cell chemotaxis antimicrobial humoral immune response mediat...
monocyte chemotaxis
lymphocyte chemotaxis
T cell migration
$T$ cell chemotaxis antimicrobial humoral immune response mediat...
monocyte chemotaxis
lymphocyte chemotaxis
T cell migration
$T$ cell chemotaxis regulation of cAMP-mediated signaling positive regulation of cAMP-mediated signaling positive regulation of calcium ion transmembra... positive regulation of calcium ion transport in..
regulation of release of sequestered calcium $\mathrm{i}$. killing of cells of other organism antimicrobial humoral response disruption of cells of other organism 1 cell chemotaxis leukocyte chemotaxis myeloid leukocyte migration
regulanon of leukocyte migration
antimicrobial humoral immune response mediat...
positive regulation of leukocyte migration
Treell migration 1
granulocyte migration myeloid leukocyte migration
regulanon of leukocyte migration
antimicrobial humoral immune response mediat...
positive regulation of leukocyte migration
Treell migration 1
granulocyte migration granulocyte migratiōn regulation of feukocyre chemotaxis regulation of leukocyle chemotaxis
chemokine-mediated signaling pathway positive regulation of leukocyte chemotaxis regulation of neutrophil migration T cell chemotaxis 1 . neutrophil migration neutrophil chemotaxis
regulation of granulocyte chemotaxis positive regulation of neutrophil migration positive regulation of neutrophil migration
ve regulation of cAMP-mediated signaling 1 . positive regulation of granulocyte chiemotaxis positive regulation of neutrophil chemotaxis

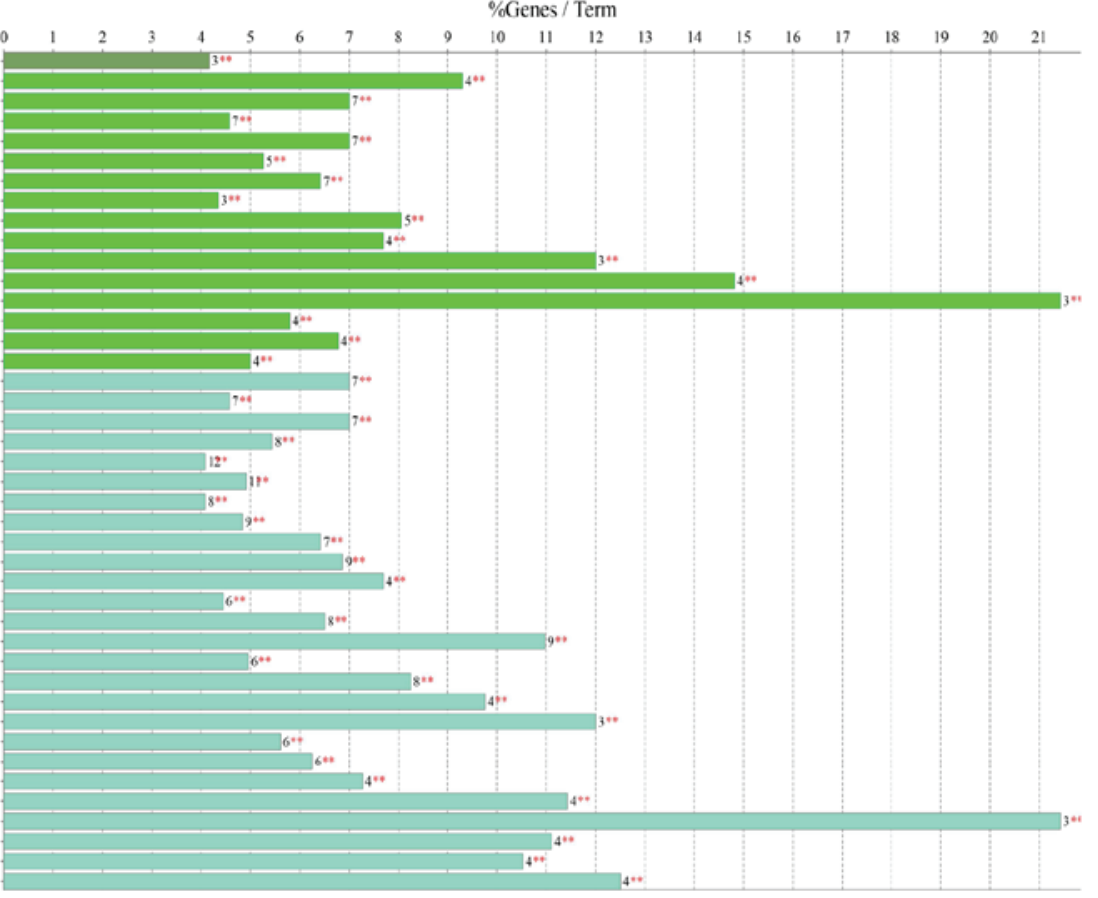

Figure 5. The top module of the protein-protein interaction network of the overlapping DEGs between ulcerative colitis and Crohn's disease. (A) The top module of the overlapping DEGs. (B) Overview chart with functional groups including specific terms for the genes involved in the top module. The percentage of terms per groups is presented. (C) Functional distribution of Gene Ontology (GO) terms in the category biological process for the genes involved in the top module. The bars represent the number of genes associated with the biological functions. The percentage of genes per term is presented as a bar label. ${ }^{* *} \mathrm{P}<0.01$; the numbers on the x-axis indicate the number of genes associated with biological functions. DEG, differentially expressed gene.

A

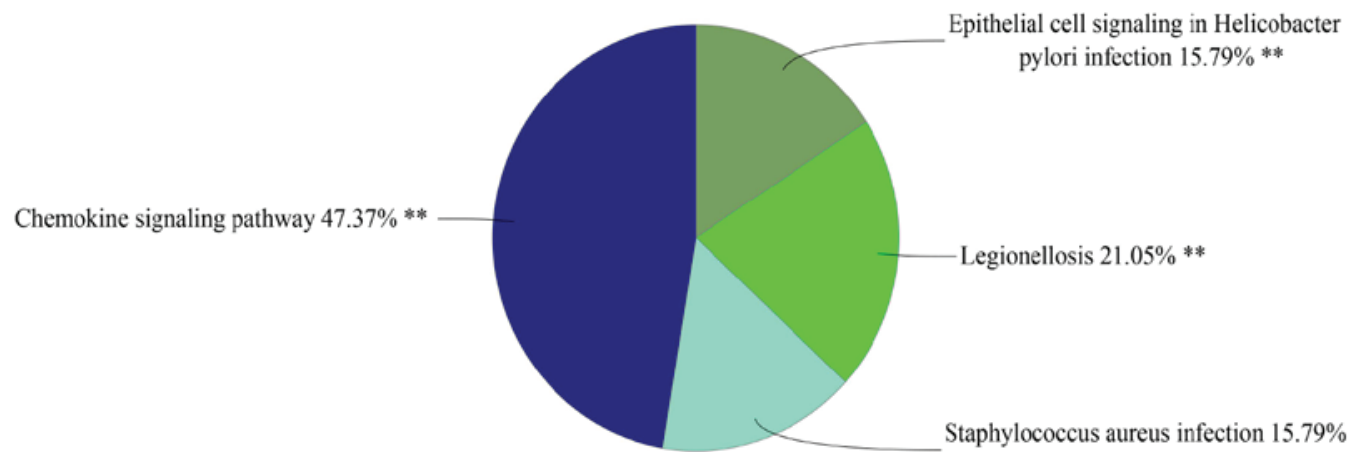

lial cell signaling in Helicobacter

**

B

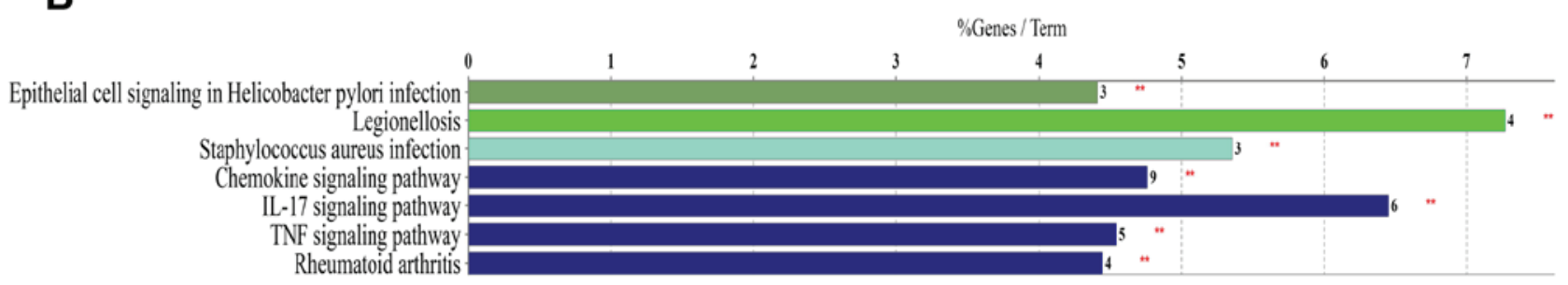

Figure 6. Functional distribution of Kyoto Encyclopedia of Genes and Genomes (KEGG) pathway terms for the top module of the protein-protein interaction network of the overlapping differentially expressed genes between ulcerative colitis and Crohn's disease. (A) Overview chart with functional groups including pathway terms for the genes involved in the top module. The percentage of terms per groups is presented. (B) KEGG pathway terms for the genes involved in the top module. The bars represent the number of genes associated with KEGG pathway terms. The percentage of genes per term is presented as a bar label ${ }^{* *} \mathrm{P}<0.01$; the numbers on the $\mathrm{X}$-axis indicate the number of genes associated with the biological pathways. 
common outcome of IBDs and may occur in UC as well as in $\mathrm{CD}$, but is more prevalent in CD $(39,40)$. Intestinal fibrosis is characterized by abnormal deposition of ECM proteins and involves collagen catabolic processes, ECM organization and ECM-receptor interaction (41), which is consistent with the results of the present study. Therefore, further investigating these biological processes and pathways may aid in gaining a better understanding of the molecular mechanisms of UC and CD.

The gene expression profile of GSE75214 analyzed in the present study was submitted by Vancamelbeke et al (18). However, the significant DEGs in the colon of patients with UC and patients with CD vs. controls and the enrichment analysis of DEGs in the present study were inconsistent with the results of the original study. There may be two reasons for these differences. One reason is that the original study had a different aim, namely to take an in-depth view on the genetic and transcriptomic bases of intestinal epithelial barrier defects in IBD, so its major focus was to evaluate the intestinal epithelial barrier, and the results were filtered from the genome-wide comparative analyses for the gene probe sets representing the selected barrier genes (18). By contrast, the aim of the present study was to identify genetic signatures in patients with IBDs and elucidate the potential associated molecular mechanisms underlying IBD subtypes, so the microarray dataset was used to obtain general genetic alterations in UC and CD patients vs. controls. The other reason is that the research objectives were different. The original study investigated the expression levels of barrier genes using colonic or ileal mucosal tissue from patients with UC and CD (active and inactive status) (18), whereas the present study only evaluated genetic alterations in colonic mucosa of patients with active IBD from the data of the original study. The abovementioned points are likely to be accountable for this difference.

Clinical discrimination between UC and CD has long been a problem due to a substantial overlap not only in pathological characteristics but also in the clinical symptoms. Therefore, it is necessary to identify discriminative key genes that may be used for diagnostics and screening of IBD subtypes. Subsequently, the respective PPI networks for IBD subtypes were constructed and the potential key genes involved in the pathogenesis of UC or CD were identified. The top 10 hub genes of the UC group included IL6, IL8, GNB4, GNG11, AGT, C-X-C motif chemokine receptor 4 (CXCR4), annexin A1 (ANXA1), phosphoinositide-3-kinase regulatory subunit 3 (PIK3R3), serum amyloid A1 and CCR7, which were mainly associated with inflammatory response. Among these genes, GNG11, GNB4, AGT, PIK3R3 and CCR7 were highly expressed in UC but not in CD. These hub genes were disease-specific, and may be used as biomarkers for differential diagnosis of UC and CD. Furthermore, the identified hub genes GNB4, GNG11 and AGT have not been previously reported to be associated with IBD. GNB4 is classified as a G protein that has a crucial role in the insulin signaling pathway (42). Incessant activation of $\mathrm{G}$ proteins (polymorphism) results in insulin resistance and ultimately increases hepatic glucose output (43). An increasing number of studies have indicated that non-alcoholic fatty liver disease (NAFLD) is common in IBDs $(44,45)$. A recent study reported that NAFLD may be detected in up to $33.6 \%$ of IBD patients, frequently in the absence of metabolic risk factors (46). Therefore, it may be speculated that GNB4 has a vital role in IBD with NAFLD. GNG11 belongs to the G protein gamma family and has a role in the transmembrane signaling system and cellular senescence $(47,48)$. A previous study reported that GNG11 was upregulated in ankylosing spondylitis, which was involved in immune system processes (49). Another study indicated that expression of GNG11 induced the generation of reactive oxygen species (50). Therefore, GNG11 may have a role in UC via the immune response and oxidative stress. Angiotensinogen (AGT) is involved in the production of angiotensin II, which is the major mediator of the action of the rennin-angiotensin system (RAS) (51), whereas the RAS mediates the regulation of sodium homeostasis, blood pressure and inflammation (52). Overexpression of AGT activates the Jak2/STAT3 signaling pathway in cells, leading to inflammation (53). Combined with the present results, the above information allows for the speculation that AGT has a role in the pathogenesis of IBD via this signaling pathway.

In addition, the hub genes of the CD group overlapped with the DEGs in the UC group, in which there were no specific hub genes. Most of the hub genes in CD were mainly associated with the chemokine signaling pathway, leukocyte migration and intestinal fibrosis-associated pathways. Based on these results, UC and CD exhibited significant differences regarding several hub genes and biological pathways. Several other hub genes may be potential therapeutic targets for IBDs. For instance, ANXA1 is a phospholipid-binding protein with potent anti-inflammatory activities (54). A previous study reported that the antagonist of ANXA1 receptors exacerbated the colitis outcome in TNF receptor $1^{-/-}$mice (55). Another study revealed that ANXA1 has a critical role in the regulation of mucosal regeneration and healing (56). These results suggest that ANXA1 may serve a pivotal role in early recovery from colitis. IL-8 is one of the major mediators of the inflammatory response in IBD. IL-8 functions as an important chemoattractant for neutrophils and is also a potent angiogenic factor (57). IL-8 is a key mediator in colonic inflammation, and is closely associated with disease activity of IBDs (58). Therefore, the increased expression of IL-8 may initiate or perpetuate IBDs. Recent study demonstrated that antibody against IL-8 effectively neutralizes IL-8-dependent neutrophil activation and migration in inflammatory diseases (59). Collectively, these hub genes may serve as promising candidates for targeted biological therapy.

Although UC and CD are different diseases, they share similar pathological characteristics. It may be useful to elucidate the shared genetic characteristics and biological pathways belonging to UC and CD. The hub genes of the overlapping DEGs in UC and CD were also identified. These hub genes were mainly enriched in pathways associated with inflammation and ECM organization. Therefore, it may be speculated that these hub genes have important functions in IBDs and have implications in the progression of these diseases. Overall, the hub genes identified constitute potential molecular targets and diagnostic biomarkers of UC or CD.

Furthermore, modules analysis of the PPI network revealed that the development of UC was associated with neuroactive 
ligand-receptor interaction and the TNF signaling pathway, whereas the pathogenesis of $\mathrm{CD}$ was also associated with the Toll-like receptor (TLR) signaling pathway. In addition, the top module analysis of the PPI network revealed that the overlapping DEGs in UC and CD were predominantly enriched in immune-associated biological processes, including chemotaxis and killing of cells of other organisms, which was consistent with the aforementioned results of the GO analysis of the present study. Furthermore, functional pathway enrichment analysis revealed that the pathogenesis of IBDs was mainly associated with chemokine signaling pathway and infectious microbes, including Staphylococcus aureus infection, legionellosis and epithelial cell signaling in Helicobacter pylori infection. Previous studies have reported a protective benefit of $H$. pylori infection against the development of IBDs (60-62). $H$. pylori may induce immune tolerance and limit inflammatory responses by TLR9 activation $(63,64)$. These results indicate the importance of $H$. pylori-induced activation of TLR9-mediated mechanisms in the pathogenesis of IBDs.

Of note, the present study has several limitations that should be emphasized. One limitation is the insufficient sample size in the dataset used for analysis. Hence, in order to achieve a more conclusive results, further analysis using a larger sample size is required. The results of comprehensive analysis combined with microarrays chips will be more reliable. Analysis of multiple transcriptomic datasets has the likelihood of discovering robust candidates for diagnosis and treatment. Therefore, it is recommended to use meta-analysis to evaluate the comprehensive effect. However, further factors also require to be taken into account. Different studies may be heterogeneous due to the variation of microarray platforms, laboratories and technicians. Furthermore, it should be ensured that the data format and class labels are consistent across datasets. In addition, due to the differences in study design, measurement errors and insufficient information [disease type (CD and $\mathrm{UC}$ ), biopsy location (colon and ileum) and disease activity status (inflamed and uninflamed)], the integrated analysis of multi-chip data may be a major challenge. A second limitation is the lack of experimental verification. In the present study, the results of a chip expression profile were analyzed using a bioinformatics method analysis and were not validated by reverse-transcription-quantitative (RT-q) PCR. Furthermore, in the original study, only the differential expression of the epithelial barrier genes MUC1, MUC4 and TFF1, CLDN1, CLDN8 and OCLN, DSG3 and MAGI1 were confirmed by RT-qPCR (18). Therefore, future studies with a larger amount of clinical samples and experimental validation are required. Despite these limitations, the present study provided novel insight into the mechanisms of IBDs.

In conclusion, the present study used an integrated analysis method to identify DEGs, as well as biological functions and pathways shared between and unique to UC and CD, thereby enhancing the current understanding of the pathogenesis of IBDs and the molecular mechanisms of UC and CD. In addition, these results may provide potential biomarkers for the differential diagnosis of UC and CD, as well as potential therapeutic targets for the development of novel IBD treatments. However, the present study only included a bioinformatics analysis and no experiments were performed to further confirm these biomarkers. Therefore, further experiments and analyses of larger datasets are required to confirm the ability of the above candidate genes in differentiating UC from CD.

\section{Acknowledgements}

The authors would like to thank Miss Juan Hua (Department of Cardiology, The Sixth Hospital of Wuhan, Affiliated Hospital of Jianghan University) for her support and language editing of this article.

\section{Funding}

The present study was supported by grants from the National Natural Science Foundation of China (grant nos. 81570486 and 81330014).

\section{Availability of data and materials}

The datasets used during the present study are available from the corresponding author upon reasonable request.

\section{Authors' contributions}

LZ and XH designed the study. CC and JT analyzed the microarray datasets and interpreted the results. JH and WQ selected and downloaded the gene expression profile from the GEO. CC wrote and edited the manuscript. All authors read the content and approved the final version.

\section{Ethics approval and informed consent}

Not applicable.

\section{Patient consent for publication}

Not applicable.

\section{Competing interests}

The authors declare that they have no competing interests.

\section{References}

1. von Stein P, Lofberg R, Kuznetsov NV, Gielen AW, Persson JO, Sundberg R, Hellstrom K, Eriksson A, Befrits R, Ost A and von Stein OD: Multigene analysis can discriminate between ulcerative colitis, Crohn's disease, and irritable bowel syndrome. Gastroenterology 134: 1869-1881; quiz 2153-2164, 2008.

2. Feuerstein JD and Cheifetz AS: Ulcerative colitis: Epidemiology, diagnosis, and management. Mayo Clin Proc 89: 1553-1563, 2014

3. Feuerstein JD and Cheifetz AS: Crohn disease: Epidemiology, diagnosis, and management. Mayo Clin Proc 92: 1088-1103, 2017.

4. Kaplan GG and Ng SC: Understanding and preventing the global increase of inflammatory bowel disease. Gastroenterology 152: 313-321.e2, 2017

5. Ng SC, Tang W, Ching JY, Wong M, Chow CM, Hui AJ, Wong TC, Leung VK, Tsang SW, Yu HH, et al: Incidence and phenotype of inflammatory bowel disease based on results from the Asia-pacific Crohn's and colitis epidemiology study. Gastroenterology 145: 158-165.e2, 2013.

6. Ng SC, Kaplan G, Banerjee R, Wei SC, Tang W, Zeng Z Chen MH, Yang H, Silva JD, Niriella MA, et al: 78 incidence and phenotype of inflammatory bowel disease from 13 countries in Asia-Pacific: Results from the Asia-Pacific Crohn's and colitis epidemiologic study 2011-2013. Gastroenterology 150: S21, 2016. 
7. Molodecky NA, Soon IS, Rabi DM, Ghali WA, Ferris M, Chernoff G, Benchimol EI, Panaccione R, Ghosh S, Barkema HW and Kaplan GG: Increasing incidence and prevalence of the inflammatory bowel diseases with time, based on systematic review. Gastroenterology 142: 46-54.e42; quiz e30, 2012.

8. Hoivik ML, Bernklev T and Moum B: Need for standardization in population-based quality of life studies: A review of the current literature. Inflamm Bowel Dis 16: 525-536, 2010.

9. Kulasingam V and Diamandis EP: Strategies for discovering novel cancer biomarkers through utilization of emerging technologies. Nat Clin Pract Oncol 5: 588-599, 2008.

10. Serna E, Morales JM, Mata M, Gonzalez-Darder J, San Miguel T, Gil-Benso R, Lopez-Gines C, Cerda-Nicolas M and Monleon D: Gene expression profiles of metabolic aggressiveness and tumor recurrence in benign meningioma. PLoS One 8: e67291, 2013.

11. Dai J, Ma Y, Chu S, Le N, Cao J and Wang Y: Identification of key genes and pathways in meningioma by bioinformatics analysis. Oncol Lett 15: 8245-8252, 2018.

12. Li XL, Zhou CY, Sun Y, Su ZY, Wang X, N Jia E, Zhang Q Jiang XF, Qi WQ and Xu Y: Bioinformatic analysis of potential candidates for therapy of inflammatory bowel disease. Eur Rev Med Pharmacol Sci 19: 4275-4284, 2015.

13. Feng J, Gao Q, Liu Q, Wang F, Lin X, Zhao Q, Liu J and Li J: Integrated strategy of differentially expressed genes associated with ulcerative colitis. Mol Med Rep 16: 7479-7489, 2017.

14. Fujimoto K, Kinoshita M, Tanaka H, Okuzaki D, Shimada Y, Kayama H, Okumura R, Furuta Y, Narazaki M, Tamura A, et al: Regulation of intestinal homeostasis by the ulcerative colitis-associated gene RNF186. Mucosal Immunol 10: 446-459, 2017.

15. Van der Goten J, Vanhove W, Lemaire K, Van Lommel L, Machiels K, Wollants WJ, De Preter V, De Hertogh G, Ferrante M, Van Assche G, et al: Integrated miRNA and mRNA expression profiling in inflamed colon of patients with ulcerative colitis. PLoS One 9: e116117, 2014.

16. UK IBD Genetics Consortium, Barrett JC, Lee JC, Lees CW, Prescott NJ, Anderson CA, Phillips A, Wesley E, Parnell K. Zhang $\mathrm{H}$, et al: Genome-wide association study of ulcerative colitis identifies three new susceptibility loci, including the HNF4A region. Nat Genet 41: 1330-1334, 2009.

17. Jostins L, Ripke S, Weersma RK, Duerr RH, McGovern DP, Hui KY, Lee JC, Schumm LP, Sharma Y, Anderson CA, et al: Host-microbe interactions have shaped the genetic architecture of inflammatory bowel disease. Nature 491: 119-124, 2012

18. Vancamelbeke M, Vanuytsel T, Farré R, Verstockt S, Ferrante M, Van Assche G, Rutgeerts P, Schuit F, Vermeire S, Arijs I and Cleynen I: Genetic and transcriptomic bases of intestinal epithelial barrier dysfunction in inflammatory bowel disease. Inflamm Bowel Dis 23: 1718-1729, 2017

19. Baumgart DC and Carding SR: Inflammatory bowel disease: Cause and immunobiology. Lancet 369: 1627-1640, 2007.

20. Albertson DG and Pinkel D: Genomic microarrays in human genetic disease and cancer. Hum Mol Genet $12 \mathrm{Spec}$ No 2 : R145-R152, 2003

21. Forini F, Nicolini G, Kusmic C, D'Aurizio R, Rizzo M Baumgart M, Groth M, Doccini S, Iervasi G and Pitto L: Integrative analysis of differentially expressed genes and miRNAs predicts complex T3-mediated protective circuits in a rat model of cardiac ischemia reperfusion. Sci Rep 8: 13870, 2018.

22. Cecchini MJ, Hosein K, Howlett CJ, Joseph M and Mura M: Comprehensive gene expression profiling identifies distinct and overlapping transcriptional profiles in non-specific interstitial pneumonia and idiopathic pulmonary fibrosis. Respir Res 19: $153,2018$.

23. Tatiya-Aphiradee $\mathrm{N}$, Chatuphonprasert $\mathrm{W}$ and Jarukamjorn $\mathrm{K}$ : Immune response and inflammatory pathway of ulcerative colitis. J Basic Clin Physiol Pharmacol 30: 1-10, 2018.

24. Strober W and Fuss IJ: Proinflammatory cytokines in the pathogenesis of inflammatory bowel diseases. Gastroenterology 140 1756-1767, 2011

25. Deng X, Szabo S, Khomenko T, Tolstanova G, Paunovic B, French SW and Sandor Z: Novel pharmacologic approaches to the prevention and treatment of ulcerative colitis. Curr Pharm Des 19: 17-28, 2013

26. Mateescu RB, Bastian AE, Nichita L, Marinescu M, Rouhani F, Voiosu AM, Benguş A, Tudoraşcu DR and Popp CG: Vascular endothelial growth factor-key mediator of angiogenesis and promising therapeutical target in ulcerative colitis. Rom J Morphol Embryol 58: 1339-1345, 2017.
27. Danese S, Sans M, de la Motte C, Graziani C, West G, Phillips MH, Pola R, Rutella S, Willis J, Gasbarrini A and Fiocchi C: Angiogenesis as a novel component of inflammatory bowel disease pathogenesis. Gastroenterology 130: 2060-2073, 2006.

28. Hu G and Agarwal P: Human disease-drug network based on genomic expression profiles. PLoS One 4: e6536, 2009.

29. Bernstein CN, Blanchard JF, Rawsthorne P and Yu N: The prevalence of extraintestinal diseases in inflammatory bowel disease: A population-based study. Am J Gastroenterol 96: 1116-1122, 2001.

30. Veloso FT: Extraintestinal manifestations of inflammatory bowel disease: Do they influence treatment and outcome? World J Gastroenterol 17: 2702-2707, 2011.

31. Kappelman MD, Galanko JA, Porter CQ and Sandler RS Association of paediatric inflammatory bowel disease with other immune-mediated diseases. Arch Dis Child 96: 1042-1046, 2011.

32. Dinca M, Fries W, Luisetto G, Peccolo F, Bottega F, Leone L, Naccarato R and Martin A: Evolution of osteopenia in inflammatory bowel disease. Am J Gastroenterol 94: 1292-1297, 1999.

33. Schulte C, Dignass AU, Mann K and Goebell H: Reduced bone mineral density and unbalanced bone metabolism in patients with inflammatory bowel disease. Inflamm Bowel Dis 4: 268-275, 1998.

34. Ciucci T, Ibáñez L, Boucoiran A, Birgy-Barelli E, Pène J, Abou-Ezzi G, Arab N, Rouleau M, Hébuterne X, Yssel H, et al: Bone marrow Th17 TNFa cells induce osteoclast differentiation, and link bone destruction to IBD. Gut 64: 1072-1081, 2015.

35. Fresno Vara JA, Casado E, de Castro J, Cejas P, Belda-Iniesta C and González-Barón M: PI3K/Akt signalling pathway and cancer. Cancer Treat Rev 30: 193-204, 2004.

36. Wu XF, Xu R, Ouyang ZJ, Qian C, Shen Y, Wu XD, Gu YH, $\mathrm{Xu} \mathrm{Q}$ and Sun Y: Beauvericin ameliorates experimental colitis by inhibiting activated $\mathrm{T}$ cells via downregulation of the PI3K/Akt signaling pathway. PLoS One 8: e83013, 2013.

37. Chen Q, Duan X, Fan H, Xu M, Tang Q, Zhang L, Shou Z, Liu X, Zuo D, Yang J, et al: Oxymatrine protects against DSS-induced colitis via inhibiting the PI3K/AKT signaling pathway. Int Immunopharmacol 53: 149-157, 2017.

38. Wei J and Feng J: Signaling pathways associated with inflammatory bowel disease. Recent Pat Inflamm Allergy Drug Discov 4: 105-117, 2010.

39. Latella G, Di Gregorio J, Flati V, Rieder F and Lawrance IC: Mechanisms of initiation and progression of intestinal fibrosis in IBD. Scand J Gastroenterol 50: 53-65, 2015.

40. Latella G, Sferra R, Speca S, Vetuschi A and Gaudio E: Can we prevent, reduce or reverse intestinal fibrosis in IBD? Eur Rev Med Pharmacol Sci 17: 1283-1304, 2013.

41. Rieder F and Fiocchi C: Intestinal fibrosis in IBD-a dynamic, multifactorial process. Nat Rev Gastroenterol Hepatol 6: 228-235, 2009

42. Saddala MS, Lennikov A, Grab DJ, Liu GS, Tang S and Huang H: Proteomics reveals ablation of PlGF increases antioxidant and neuroprotective proteins in the diabetic mouse retina. Sci Rep 8: 16728, 2018.

43. Benjafield AV, Lin RC, Dalziel B, Gosby AK, Caterson ID and Morris BJ: G-protein beta3 subunit gene splice variant in obesity and overweight. Int J Obes Relat Metab Disord 25: 777-780, 2001.

44. Principi M, Iannone A, Losurdo G, Mangia M, Shahini E, Albano F, Rizzi SF, La Fortezza RF, Lovero R, Contaldo A, et al: Nonalcoholic fatty liver disease in inflammatory bowel disease: Prevalence and risk factors. Inflamm Bowel Dis 24: 1589-1596, 2018.

45. Sartini A, Gitto S, Bianchini M, Verga MC, Di Girolamo M, Bertani A, Del Buono M, Schepis F, Lei B, De Maria N and Villa E: Non-alcoholic fatty liver disease phenotypes in patients with inflammatory bowel disease. Cell Death Dis 9: 87, 2018.

46. Bessissow T, Le NH, Rollet K, Afif W, Bitton A and Sebastiani G: Incidence and predictors of nonalcoholic fatty liver disease by serum biomarkers in patients with inflammatory bowel disease. Inflamm Bowel Dis 22: 1937-1944, 2016.

47. Balcueva EA, Wang Q, Hughes H, Kunsch C, Yu Z and Robishaw JD: Human G protein gamma(11) and gamma(14) subtypes define a new functional subclass. Exp Cell Res 257: $310-319,2000$ 
48. Hossain MN, Sakemura R, Fujii M and Ayusawa D: G-protein gamma subunit GNG11 strongly regulates cellular senescence. Biochem Biophys Res Commun 351: 645-650, 2006.

49. Fang F, Pan J, Xu L, Li G and Wang J: Identification of potential transcriptomic markers in developing ankylosing spondylitis: A meta-analysis of gene expression profiles. Biomed Res Int 2015: 826316, 2015.

50. Takauji Y, Kudo I, En A, Matsuo R, Hossain MN, Nakabayashi K, Miki K, Fujii M and Ayusawa D: GNG11 (G-protein subunit $\gamma$ 11) suppresses cell growth with induction of reactive oxygen species and abnormal nuclear morphology in human SUSM-1 cells. Biochem Cell Biol 95: 517-523, 2017.

51. Farcas A, Gligor F, Bucsa C, Mogosan C, Bojită M and Dumitrașcu D: The current insight on dual renin-angiotensin system blockade: A data review with a focus on safety. Farmacia 63: 325-333, 2015.

52. Raizada V, Skipper B, Luo W and Griffith J: Intracardiac and intrarenal renin-angiotensin systems: Mechanisms of cardiovascular and renal effects. J Investig Med 55: 341-359, 2007.

53. Shen L, Zhang T and Lu H: Overexpression of AGT promotes bronchopulmonary dysplasis via the JAK/STAT signal pathway. Oncotarget 8: 96079-96088, 2017.

54. Dalli J, Norling LV, Renshaw D, Cooper D, Leung KY and Perretti M: Annexin 1 mediates the rapid anti-inflammatory effects of neutrophil-derived microparticles. Blood 112: 2512-2519, 2008.

55. Sena AA, Pedrotti LP, Barrios BE, Cejas H, Balderramo D, Diller A and Correa SG: Lack of TNFRI signaling enhances annexin A1 biological activity in intestinal inflammation. Biochem Pharmacol 98: 422-431, 2015.

56. Babbin BA, Lee WY, Parkos CA, Winfree LM, Akyildiz A Perretti $\mathrm{M}$ and Nusrat A: Annexin I regulates SKCO-15 cell invasion by signaling through formyl peptide receptors. J Biol Chem 281: 19588-19599, 2006.

57. Yang XD, Corvalan JR, Wang P, Roy CM and Davis CG: Fully human anti-interleukin-8 monoclonal antibodies: Potential therapeutics for the treatment of inflammatory disease states. J Leukoc Biol 66: 401-410, 1999.
58. Skov L, Beurskens FJ, Zachariae CO, Reitamo S, Teeling J, Satijn D, Knudsen KM, Boot EP, Hudson D, Baadsgaard O, et al: IL-8 as antibody therapeutic target in inflammatory diseases: Reduction of clinical activity in palmoplantar pustulosis. J Immunol 181: 669-679, 2008.

59. Mitsuyama K, Toyonaga A, Sasaki E, Watanabe K, Tateishi H, Nishiyama T, Saiki T, Ikeda H, Tsuruta O and Tanikawa K: IL-8 as an important chemoattractant for neutrophils in ulcerative colitis and Crohn's disease. Clin Exp Immunol 96: 432-436, 1994.

60. Luther J, Dave M, Higgins PD and Kao JY: Association between Helicobacter pylori infection and inflammatory bowel disease: A meta-analysis and systematic review of the literature. Inflamm Bowel Dis 16: 1077-1084, 2010.

61. Rokkas T, Gisbert JP, Niv Y and O'Morain C: The association between Helicobacter pylori infection and inflammatory bowel disease based on meta-analysis. United European Gastroenterol J 3: 539-550, 2015.

62. Wu XW,Ji HZ, Yang MF, Wu L and Wang FY: Helicobacter pylori infection and inflammatory bowel disease in Asians: A meta-analysis. World J Gastroenterol 21: 4750-4756, 2015.

63. Varga MG, Piazuelo MB, Romero-Gallo J, Delgado AG, Suarez G, Whitaker ME, Krishna US, Patel RV, Skaar EP, Wilson KT, et al: TLR9 activation suppresses inflammation in response to Helicobacter pylori infection. Am J Physiol Gastrointest Liver Physiol 311: G852-G858, 2016.

64. Owyang SY, Luther J, Owyang CC, Zhang M and Kao JY: Helicobacter pylori DNA's anti-inflammatory effect on experimental colitis. Gut microbes 3: 168-171, 2012.

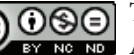

$$
\begin{aligned}
& \text { This work is licensed under a Creative Commons } \\
& \text { Attribution-NonCommercial-NoDerivatives } 4.0 \\
& \text { International (CC BY-NC-ND 4.0) License. }
\end{aligned}
$$

